\title{
ODONTOGENIC MYXOMA VERSUS SOFT TISSUE MYXOMA: IMMUNOHISTOCHEMICAL STUDY
}

\author{
Shymaa Ahmed Hamza*, Samia Mostafa El-Azab* and Safa Fathy Abd El-Ghani*
}

\begin{abstract}
Background: Odontogenic Myxoma (OM) and Soft Tissue Myxoma (STM) are two myxoid tumors that may arise in the oral cavity. They often present a diagnostic and treatment challenge because of the histological overlap at the light microscopial level. Our study aimed at understanding their origin and biological behavior through comparing the immunohistochemical expression level of the neural stem cell marker; Nestin (N), as well as the invasion marker; Matrix Metalloprotienase 9 (MMP-9).
\end{abstract}

Methods: The N and MMP-9 expression area percent, were measured and calculated in a total of 16 Myxoma specimens, where 9 cases of OM and 7 cases of STM were examined.

Results: N and MMP-9 were positively expressed in both OM and STM showing maily cytoplasmic immunostaing, where higher expression level in $\mathrm{OM}$ was found; using both markers.

Conclusions: Finally, our results were able to suggest the common origin for OM and STM to be the odontogenic ectomesenchyme which is derived from neural crest cells. We propose that STM might be considered the peripheral entity of OM. Moreover, OM is less differentiated and more aggressive than STM.

KEY WORDS: Nestin, MMP-9, Odontogenic Myxoma, Soft Tissue Myxoma.

\section{INTRODUCTION}

Myxoid tumors are mesenchymal tumors characterized by the presence of abundant myxoid extracellular matrix (ECM). The behavior of myxoid tumors ranges from truely benign to frankly malignant with metastatic potential ${ }^{(1)}$. OM and STM are two examples of myxoid tumors that may arise in the oral cavity. While OM is a common benign odontogenic tumor occurring in the jaws ${ }^{(2,3)}$, STM is a rare lesion that can occur anywhere owing to its primitive cell origin ${ }^{(4)}$.

The differential diagnosis of myxomatous tumors can be very challenging because of the significant histological overlap between the different entities at the light microscopical level (1). Integration of histopathological examination with extensive additional clinical and radiographic data is essential. Moreover, evaluation of the immunohistochemical

* Department of Oral and Maxillofacial Pathology, Faculty of Oral and Dental Medicine, Cairo University. 
expression of molecular biomarkers in these lesions may advance the general understanding of the origin, behavior, prognosis and proper treatment of myxomatous tumors ${ }^{(1,5)}$. N and MMP-9 are two widely used biomarkers. However, very few studies were accomplished to examine their expression in myxomatous tumors ${ }^{(6,7}$ and 8$)$.

$\mathrm{N}$ is one of the intermediate filaments constituting the cytoskeleton, it can be used as a marker of neural stem cells or progenitor cells. Also, it is closely related to tooth development and repair of dentin. Although human $\mathrm{N}$ expression is present during tooth development, it disappears when development is complete. Furthermore, its expression has been reported in other pathological conditions, such as central and peripheral nervous system tumors ${ }^{(9)}$. On the other hand, MMP-9 is one of the extracellular endopeptidases involved in degradation and remodeling of ECM. MMPs are known to be involved in the progression and invasiveness of many types of tumors ${ }^{(9,10)}$.

The aim of the our work was to detect the immunohistochemical expression of $\mathrm{N}$ and MMP9 in both OM and STM, compare the expression levels of these two markers between both tumors and finally to correlate the obtained results with the biological behaviors of both tumors.

\section{MATERIAL AND METHODS}

\section{Tissue Specimens}

Specimens of OM and STM were retrieved from the archival paraffin blocks of Oral Pathology Departments, Faculty of Oral and Dental Medicine, Cairo University and Alexandria University, as well as from the files of Surgical Pathology Department, National Cancer Institute, Cairo University. All the cases diagnosed from 2002 to 2014 were included to be used in this study, where 9 cases of $\mathrm{OM}$ and 7 cases of STM were examined.

\section{Histopathological Examination}

A $5 \mu$ thick section from each specimen was cut and stained by routine haematoxylin and eosin $(H \& E)$ to re-evaluate the diagnosis under ordinary light microscope. The most representative sections were selected for immunostaining.

\section{Immunohistochemistry}

In the current work, the streptavidin biotin technique was carried out. This is a 3- step technique which has an unconjugated primary antibody as the first layer, followed by a biotinylated secondary antibody. The third layer is a horse radish peroxidise enzyme-labelled streptavidin.

The positive immune-reaction of N and MMP-9 were detected as a brownish colour in cytoplasm, and it was assessed by two methods:

Ordinary Light Microscope: The ordinary light microscope was used to detect the positive and negative immunostaining as well as localization of the positive reaction within the tissues.

Image Analyzer Computer System: The immunostained sections were examined using Leica Quin 500 analyzer computer system (Germany). The image analyzer was calibrated automatically to convert the measurement units (pixels) produced by the image analyzer program into actual micrometer units.

The immunoreactivity was measured as area and area percent in a standard measuring frame of area 38883.69 micrometer $^{2}$ per 10 fields using magnification $(x 400)$ by light microscopy transferred to the screen. The areas containing the most uniformly stained tissues were chosen for evaluation. These areas were masked by a blue binary colour to be measured by the computer system. Mean values were obtained for all the specimens.

\section{Statistical Analysis}

The data obtained from the computer image analysis was tabulated and statistically analysed. 
Data was presented as mean and standard deviation (SD) values. One-way Analysis of Variance (ANOVA) was used for the comparisons. Student's $t$ test was used for the comparisons. $\mathrm{P}$ value was considered significant at $95 \%$ confidence interval when $\mathrm{p} \leq 0.05$.

\section{RESULTS}

\section{Immunoreaction to Nestin}

In OM, $N$ was found in all cases (100\%) with variable amounts of expressivity ranging from moderate to strong immunoreactions (figs.3). It was mainly localized to the cytoplasm and cytoplasmic processes of stellate tumor cells, however some cases (3/9) showed nuclear reaction. Also, positive immunoreaction was observed in the cytoplasmic processes of the stellate cells (fig. 3)

In STM cases, $80 \%$ of the studied specimens showed positive $\mathrm{N}$ immunoreactivity. It was localized to the cytoplasm of the tumor cells and its cytoplasmic extensions (fig. 4). Nuclear immunoreaction was also detected in 2 out of 7 cases. In addition, positive membranous reaction was noted in the surface epithelium in $100 \%$ of the examined specimens (fig. 4). It was also observed that $20 \%$ of the studied cases showed negative reaction.

\section{Immunoreaction to MMP-9}

All the studied cases (100\%) of OM showed moderate to strong positive MMP-9 immunoreactivity (fig. 5). Cytoplasmic and nuclear staining patterns were acquired by the anastomosing tumor cells (fig.5). Also positive reaction was seen in the cytoplasmic extensions and obvious negative reaction was noted in connective tissue matrix.

Similarly, in STM specimens, positive MMP-9 immunoexpression was noted in all cases (100\%). Moderate to strong cytoplasmic immunoreactivety was observed in both the cytoplasm and the nucleus of tumor cells (fig. 6). Also, membranous reaction was acquired by the surface epithelium .

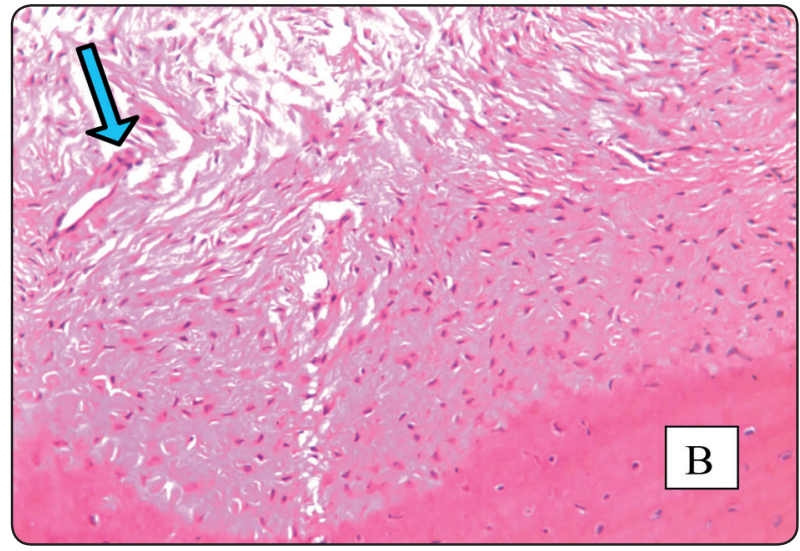

Fig (1) A photomicrograph of OM showing stellate cells lying in a myxoid matrix nearby bone trabeculae (B). Giant cells are also noted (arrow), H\&E, X200

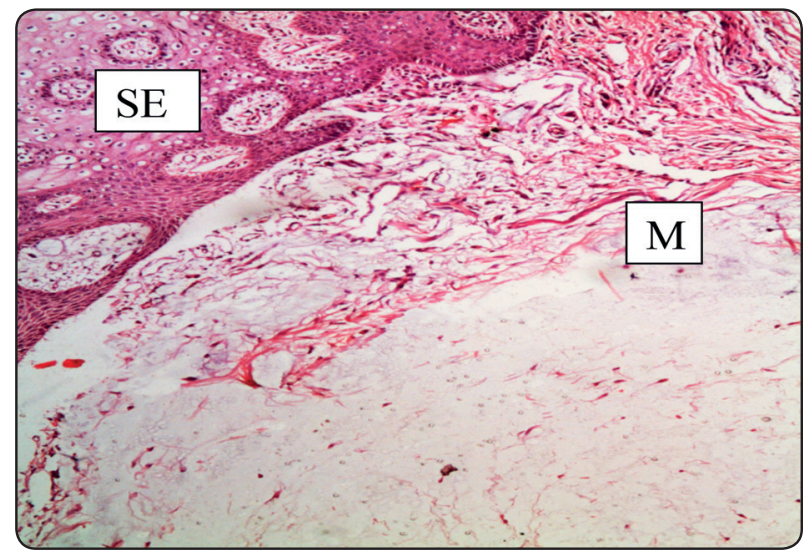

Fig (2) A photomicrograph of STM showing stellate cells interspersed in myxoid matrix (M) and covered with surface epithelium (SE), H\&E, x100.

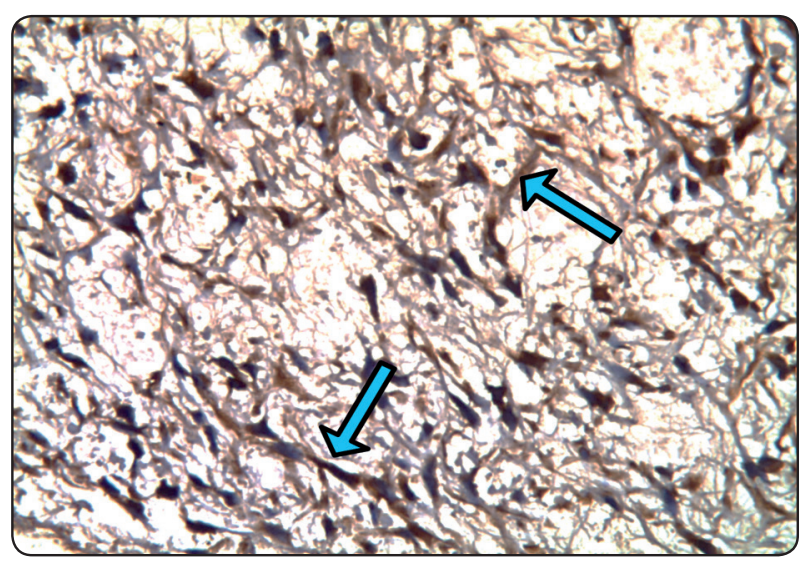

Fig (3) A photomicrograph of OM showing diffuse strong $\mathrm{N}$ immunoreactivity in stellate cells. Note: the positive reaction in the cytoplasmic extentions (arrows) anti-N antibody, $\mathrm{x} 400$. 


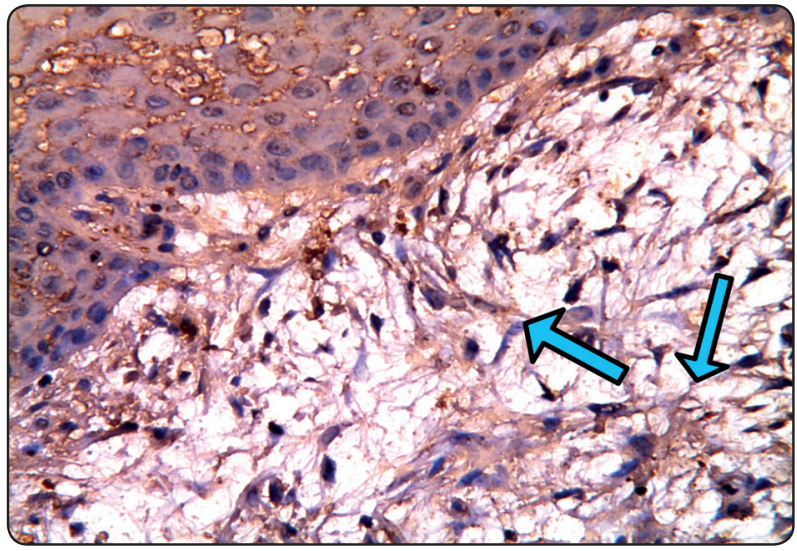

Fig (4) A higher magnification of the previous case shows positive $\mathrm{N}$ cytoplasmic localization in the tumor cells and their cytoplasmic extensions. Some tumor cells are showing nuclear $\mathrm{N}$ immunoreaction (arrows), anti-N antibody, $\mathrm{x} 400$.

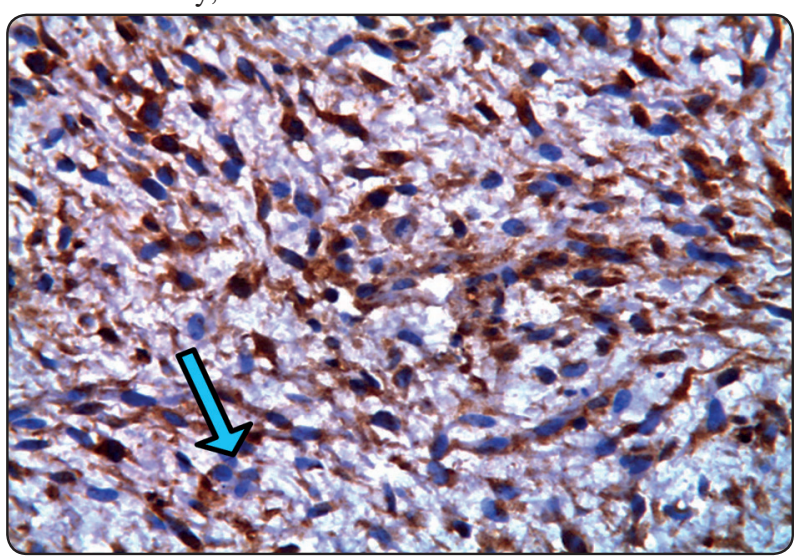

Fig (5) A photomicrograph of $\mathrm{OM}$ showing strong positive MMP-9 cytoplasmic localization in spindle cells. Note: the negative nuclear reaction in most of the tumor cells (arrow), anti-MMP-9 antibody, $\mathrm{x} 400$.

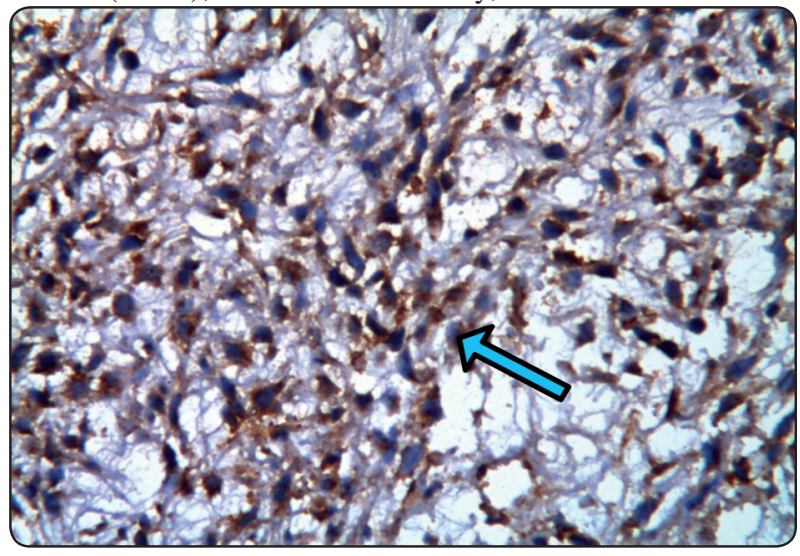

Fig (6) A higher magnification showing MMP-9 cytoplasmic and nuclear (arrow) localization in tumor stellate cells, anti-MMP-9 antibody, x400.

\section{DISCUSSION}

$\mathrm{OM}$ and STM are benign tumors occurring in the jaws and various sites in the head and neck, repectively. While, OM has high recurrence rate and tendency for bone destruction, STM has a very low rate of recurrence and a less aggressive course ${ }^{(11)}$. Their development, progression and difference in behavior, could be affected by alterations of many molecules and genes. Studying these 2 tumors would allow better understanding of their underlying molecular mechanisms, in addition to development of new therapeutic applications ${ }^{(12)}$.

Moreover, to date, little is known about the histogenesis of both tumors and for the best of our knowledge, scarce studies were found in the available literature examining OM and STM. Many lines of evidence have shown $\mathrm{N}$-positive cells to be neural stem/progenitor cells. Therefore, many researches have involved the use of $\mathrm{N}$ to detect neural stem cells ${ }^{(13)}$. Based on our results, we can propose that both OM and STM share the same origin and are derived from odontogenic ectomesenchyme which originated from the neural crest cells, as both tumors expressed $\mathrm{N}$ immunohistochemically

Although both tumors showed moderate to strong $\mathrm{N}$ immunoreactivety, the statistical analysis of our work showed that the expression of $\mathrm{N}$ was obviously higher in OM than STM. Since N is an indicator for dedifferentiation, therefore our results can propose that OM is less differentiated than STM. This low level of differentiation can also suggest a more aggressive behavior of $\mathrm{OM}$ when compared to STM. This came in accordance with Ehrmann et al. ${ }^{(14)}$ and Du et al. ${ }^{(15)}$. The later concluded that, since $\mathrm{N}$ was down-regulated or completely disappeared during differentiation, it could be used as an auxiliary indicator of dedifferentiation and progression.

Our current work showed N cytoplasmic localization in the positive cells of OM and STM. This expression pattern can be attributed to its structure, being one of the intermediate filaments constituting the cytoskeleton. This finding accords 
with Ehrmann et al. ${ }^{(14)}$ and Du et al. ${ }^{(15)}$ who studied $\mathrm{N}$ expression in glioblastoma, malignant melanoma and osteochondroma, respectively. The results of our present work showed $\mathrm{N}$ localization in the endothelial cells of blood vessels of OM. This can propose a role for $\mathrm{N}$ in the angiogenesis of the tumor. This came corresponding to Tampaki et al. ${ }^{(16)}$, who reported $\mathrm{N}$ implication in tumor angiogenesis suggesting its role in facilitating tumor growth.

Considering MMP-9, all the selected specimens showed positive MMP-9 expression in both OM and STM. These results came in line with Kumamoto ${ }^{(12)}$ who suggested that positive MMP-9 immunoreaction contributed to the local invasiveness of these tumors. The outcomes of our work also agreed with Miyagi et al. ${ }^{(8)}$, who investigated MMP-9 in tumor samples derived from OM. They observed that MMP-9 was capable to hydrolyze the components of ECM and that it could degrade type IV collagen, the major part of the basement membrane. Thus, they concluded that expression of MMP-9 is associated with the invasive phenotype of OM. Contrarily, Bast et al. (6) showed that all OM specimens, myxomatous dental follicular tissue and myxoid dental pulp were negative to MMP-9. They recommended that further work should be done to reveal other possible factors contributing for OM aggressive behavior.

Our results showed that MMP-9 immunoreactivety in $\mathrm{OM}$ was double that of STM. This can explain the difference in their clinical behaviors. This finding is in agreement with Ayctac-Yazicioglu et al. ${ }^{(1)}$, who added that $\mathrm{OM}$ aggressiveness and high recurrence rate were the results of local invasion into cancellous bone, beyond radiographically visible margins. In 2012, Mauro et al. ${ }^{(10)}$ reported the association between lack of recurrence of OM and absence of MMP-9 expression in the tumor cells. In our present work, MMP-9 expression was found to be localized in the cytoplasm of tumor cells in both OM and STM in all the studied specimens. This finding came in accordance with Ohashi et al. ${ }^{(17)}$, as they found strong immunoreaction for MMP-9 in the cytoplasm of squamous cell carcinoma cells.

Finally, our results were able to suggest the common origin for OM and STM to be the odontogenic ectomesenchyme which is derived from neural crest cells. We propose that STM might be considered the peripheral counterpart of OM. Moreover, $\mathrm{OM}$ is less differentiated and more aggressive than STM. Considering the used biological markers, while $\mathrm{N}$ could be considered as a valuable biomarker for differentiation and progression, MMP-9 is a reliable prognostic marker for progression and recurrence. However, further studies are recommended to confirm our results.

\section{REFERENCES}

1. Willems, S: Molecular genetic aspects and characterization of the extracellular matrix of myxoidtumours of soft tissue, pp. 13, 18, 23, 24, 31, 33, 34, 35, 37, 41, 64, 65, 88, Gildeprint, Enschede, The Netherlands, 2011.

2. Miyagi, S; Maranduba, C; Silva, Fde S and Marques, M: Dental pulp stem cells express proteins involved in the local invasiveness of odontogenic myxoma. Braz Oral Res., 26:139-144, 2012.

3. Munoz, A; Rodriguez, M; Molina, R; Raga, F; Hernandez, F; Morales, J; Trujillo, J; Escalera, C; Hernandez, G: The orosomucoid 1 protein (alpha1 acid glycoprotein) is overexpressed in odontogenic myxoma. Proteome Science, 10:49, 2012.

4. Regezi, J; Sciubba, J and Jordan, R: Oral Pathology: Clinical Pathological Correlations. 5th ed. pp.161, Linda Dolan, Elsevier Inc., China , 2008.

5. Manssano, J; Regater, O; Januar, G and Ferreira, A: Oral squamous cell carcinoma: Review of prognostic and predictive factors. Oral surg, Oral Med path, Oral Radiol \& Endod, 102:67-76, 2006.

6. Bast, B; Pogrel, M and Regezi, J: The expression of apoptotic proteins and matrix metalloproteinases in odontogenic myxoma. J Oral Maxillofac Surg, 61:1463-6, 2003.

7. Fujita, S; Hideshima, K and Ikeda, T: Nestin expression in odontoblasts and odontogenicectomesenchymal tissue of odontogenictumours. J Clin Pathol, 59:240-245, 2006. 
8. Miyagi, S; Hiraki, K; Martins, M and Marques, M: Expression of matrix metalloproteinases 2 and 9 in odontogenic myxoma in vivo and in vitro. J Oral Sci, 50(2):187-192, 2008.

9. Gomes, C; Diniz, D; Duarte, A; Bernardes, V: Molecular review of odontogenicmyxoma. Oral oncology, 47:325328, 2011.

10. Mauro, A; Lipari, L; Tortorici, S; Leone, A; Gerbino, A and Buscemi, M: Expression of MMP-2 and MMP-9 in odontogenic myxoma in a child: report of a clinical case. Odontology, 101(2):233-238, 2012.

11. Aytac-Yazicioglu, D; Eren, H and Gorgun, S: Peripheral odontogenic myxoma located on the maxillary gingiva: report of a case and review of literature. Oral Maxillofac Surg, 12: 167-171, 2008.

12. Kumamoto, $\mathrm{H}$ : Molecular alterations in the development and progression of odontogenic tumors. Oral Med Pathol., 14: 121-131, 2010.
13. Liu, L; Shi, M; Wang, L; Hou, S; Wu, Z; Zhao, G and Deng, Y: Ndrg2 expression in neurogenic germinal zones of embryonic and postnatal mouse brain. J Mol Histol, 43 (1):27-35, 2012.

14. Ehrmann, J; Kolar, Z and Mokry, J: Nestin as a diagnostic and prognostic marker: immunohistochemical analysis of its expression in different tumors. J Clin Pathol, 58:222$223,2005$.

15. Du, K; Peng, Y; Zhang, L; Liang, A and Huang, D: Expression of the stem cell marker nestin in pre/hypertrophic chondrocytes in osteochondroma. The J Int Med Res, 39: $348-357,2011$.

16. Tampaki, E; Nakopoulou, L; Tampakis, A; Kontzoglou, K; Weber, WP and Kouraklis, G: Nestin involvement in tissue injury and cancer - a potential tumor marker? Cell Oncol, 37(5):305-315, 2014.

17. Ohashi, K; Nemoto, T; Nakamura, K and Nemori, R: Increased expression of matrix metalloproteinase 7 and 9 and membrane type 1-matrix metalloproteinase in esophageal squamous cell carcinomas. Cancer, 88:2201-2209, 2000. 